\title{
The impact of funding changes on the implementation of primary health care policy
}

\author{
Mary P. Finlayson ${ }^{1}$, Nicolette F. Sheridan ${ }^{2}$, Jacqueline M. Cumming ${ }^{3}$ and Sandra Fowler ${ }^{4}$ \\ ${ }^{1}$ Associate Professor, School of Nursing, University of Auckland, Auckland, New Zealand \\ ${ }^{2}$ Associate Dean (Equity), School of Nursing, Faculty of Medical and Health Sciences, University of Auckland, \\ Auckland, New Zealand \\ ${ }^{3}$ Associate Professor and Director, Health Services Research Centre, Victoria University of Wellington, Wellington, \\ New Zealand \\ ${ }^{4}$ Research fellow, Health Services Research Centre, Victoria University of Wellington, Wellington, New Zealand
}

\begin{abstract}
Aim: To ascertain how new funding arrangements, introduced in New Zealand's 2001 Primary Health Care (PHC) Strategy, have impacted on the expansion of nurses' role in general practice. Background: Nurses are central to the new policy that was designed to improve the health status of New Zealanders and reduce inequalities in health. Nurses were to be a crucial part of the PHC team, expanding their current roles to provide increased access to appropriate services. This paper investigates how the new funding arrangements, introduced as part of the policy, have impacted on the expansion of nurses' roles and consequently the realisation of the policy goals. Methods: Semi-structured interviews were undertaken with 128 key stakeholders five years after the introduction of the PHC Strategy, and surveys were completed by practice nurses, general practitioners and practice managers in purposively selected practices within the 20 participating Primary Health Organisations. Findings: There has been substantial growth in the development of nursing roles for some nurses in general practice; however, this expansion has not been universal and one of the main reasons for this is the way funding devolves at the practice level. One of the consequences of the policymakers not taking into account the business model of the majority of general practices, is the resulting overarching goal of the strategy not being realised, and inequalities in health status remaining.
\end{abstract}

Key words: general practices; nurses; policy implementation; primary health care

Received 19 January 2011; accepted 18 July 2011; first published online 22 August 2011

\section{Introduction}

This paper explores New Zealand's (NZ's) experience of the impact of changes to primary health care $(\mathrm{PHC})^{1}$ funding on the expansion of

\footnotetext{
Correspondence to: Dr Nicolette F. Sheridan, School of Nursing, Faculty of Medical and Health Sciences, University of Auckland, Private Bag 92019, Auckland 1142, New Zealand. Email: n.sheridan@auckland.ac.nz

${ }^{1}$ Primary health care is the first level of contact with the health system and is integral to, and a central function of, New Zealand's health system (King, 2001).
}

the nursing role in general practice. It highlights the need for governments to take into account existing organisational structures and the interests of key stakeholders when implementing new policy.

Reforms of the NZ health system outlined in the 2001 PHC Strategy (hereafter the Strategy) sought to further develop the PHC sector to improve health status and reduce inequalities in health (King, 2001). Similar trends can be seen in England, the United States of America, Canada and Australia, as each country has focused on devolving healthcare services to community settings 
and increasing interdisciplinary work and preventive services (Bodenheimer, 2003; Armstrong, 2005; Dodoo et al., 2005; Department of Health, 2006). This shift of services to PHC has given rise to new and expanded nursing roles internationally as well as a substantial increase in the type and number of nurses providing PHC services (Carnwell and Daly, 2003; Buchan and Calman, 2005).

The NZ PHC Strategy signalled a move beyond general practitioner (GP)-driven, fee-for-service, individually focused care towards the provision of services targeted to populations. Its aims were to be achieved by enrolling patients with PHC providers and introducing new funding arrangements to improve access to services for all New Zealanders and promote a population-based approach. It promoted collaboration in service delivery and governance and suggested that a team approach be adopted to ensure a more effective and efficient way of delivering $\mathrm{PHC}$ with particular emphasis on expanding the role of nurses (King, 2001).

This paper focuses on whether and how the new funding arrangements have led to an expanded role for nurses in general practice in $\mathrm{NZ}$ and the implications for the implementation of the Strategy.

\section{Background}

Widespread health sector reforms were introduced in NZ in 2001 to create a health system that 'people can trust, that is there when they need it regardless of their ability to pay, and that really helps reduce inequalities' (King, 2001). The cornerstone of the Strategy was the establishment of Primary Health Organisations (PHOs), local notfor-profit organisations responsible for ensuring the provision of PHC services to their enrolled populations. By April 2009, there were 81 PHOs covering just over 4 million people, approximately $95 \%$ of the estimated total population of NZ (Ministry of Health, 2009e).

Prior to the Strategy, the majority of PHC services had been provided by GPs with nurses in the practices working in a support role, generally in GPowned practices. District Health Boards (DHBs) and non-governmental organisations (NGOs) have also made a major contribution to $\mathrm{PHC}$, but they are not the focus of this paper.

\section{Funding of PHC services}

The Strategy significantly changed the way PHC services were funded in NZ. Traditionally, PHC was dominated by a GP-led, privately owned business model with income derived from partial subsidies from government and fee-forservice user charges. In the new environment, PHOs receive government funding via the 21 (now 20) DHBs to subsidise a range of health services for their enrolled populations, and this is then passed down to general practices using a capitation formula. General practices are then at liberty to allocate the funding in any way they wish. The core mechanism for government funding is capitation-based subsidies in addition to fee-for-service or budgeted payments for other initiatives not funded on a capitation basis, for example, Services to Improve Access (SIA) and Care Plus (Croxon et al., 2009). In addition, practices can charge their patients' co-payments for services.

Capitation funding to PHOs subsidises the cost of visits to general practice, health promotion programmes, SIA for high-need groups and $\mathrm{PHO}$ administrative fees (Ministry of Health, 2009d). Under the capitation-based system, PHOs are paid according to the number of people enrolled in their member practices, rather than the number of times a doctor sees patients (Ministry of Health, 2009b). Factors such as age, gender, ethnicity and deprivation quintile influence the amount of subsidy a $\mathrm{PHO}$ receives.

In addition to the $\mathrm{PHO}$ capitation funding, a number of initiatives at a national level funded by the Ministry of Health have also been introduced to improve access to PHC, improve the management of chronic diseases and establish expanded and innovative PHC nursing services. These initiatives are: Reducing Inequalities Contingency Funding (RICF); Care Plus; SIA; and nursing innovation projects.

RICF concentrates on services delivered in the home or community to high need population groups. A large number of these projects involved nursing outreach initiatives that targeted specific populations and areas of need. Care Plus is a programme that requires PHOs to enrol eligible patients and provides additional funding for people who have to visit a GP or nurse more frequently because they have two or more chronic conditions or a terminal illness (Ministry of Health, 2009a). SIA funding is available for all PHOs to 
reduce inequalities among those populations that are known to have the worst health status: Māori; Pacific; and those living in NZ's highest deprivation areas. Between 2004 and 2006, funding was also allocated to support 11 PHC nursing innovations (Primary Health Care Nurse Innovation Evaluation Team, 2007). These focused on creating nursing leadership roles to develop the PHC nursing workforce and enhance service integration, and provide nursing services to populations experiencing barriers to access.

\section{Role of the nurse}

Prior to the PHC reforms, general practice and other community-based services in NZ were predominantly market-driven, with a focus on outputs. Doctors were the prominent care providers with practice nurses undertaking delegated tasks. The majority of practice nurses were not working in an expanded role or utilising a broad range of skills and few had postgraduate qualifications (Kent et al., 2005).

In the Strategy, the then Minister of Health described nurses as 'crucial' to its successful implementation, not only in assisting PHOs to collect information about enrolled populations and identifying their health needs, but also because they are particularly well placed to design and lead many population-based initiatives such as disease prevention, screening and health education programmes. The Strategy therefore presented an opportunity for PHC nurses to expand their day-to-day activities in care delivery, to get involved in the governance of PHOs, and it encouraged the development of higher level skills and knowledge.

Another important role is that of the Nurse Practitioner. The Nurse Practitioner role was introduced in NZ 10 years ago. Nurse Practitioners have master's degrees and prescribing authority and are registered to work in specific speciality areas across the primary and secondary sectors. They are ideally placed to work in PHC as they have extensive clinical and contextual knowledge of their speciality areas that enables them to respond to the health needs of their communities and provide appropriate and costeffective services. Nurse Practitioners are trained and registered to work as independent practitioners and they have the potential to enhance access to services and choice of provider, as well as develop innovative ways of reaching communities and under-served populations. In 2011, there are only 31 out of 95 Nurse Practitioners registered to work in the speciality area of PHC in NZ (Nursing Council of New Zealand, 2011).

\section{Methods}

The aim of this paper is to present the research findings into how new funding arrangements, introduced in the 2001 Strategy, have impacted on expanding the role of nurses in general practice and whether the nursing role has expanded as a result of the Strategy. To do this, it draws on the findings from the evaluation Nursing Developments in Primary Health Care 2001-2007 (Finlayson et al., 2009).

Semi-structured interviews were undertaken with practice, PHO and DHB (staff in seven DHB regions, Ministry of Health representatives, professional representatives and nurse leaders.

\section{Selection of DHBs, PHOs and general practices}

Seven DHBs were purposively selected to represent rural and urban; large and small; and North and South Island regions. Similarly, 20 PHOs from these DHBs were purposively selected from a Ministry of Health list that categorised them according to size, funding formula and focus (Māori, Pacific and other). General practices were selected on the advice of the PHO managers to represent diverse views.

\section{Interview process}

The semi-structured interviews were conducted by nine interviewers trained to use an interview guide developed by the research team and steering committee. The interview guides focussed on: management and governance; relationships between organisations; funding; workforce; team work; roles and responsibilities; remuneration; continuing education; and work satisfaction.

Typically, the interviews, lasting 1 hour, were conducted on a one-to-one basis; however, a number of interviews were conducted with two or more interviewees present. 


\section{Analysis}

Using a general inductive approach (Thomas, 2006), each interview was transcribed and the transcripts were systematically and rigorously read and coded by an independent reviewer. The codes were then reduced into themes by the multidisciplinary research team. The emergent themes that this paper focused on were: expanding services; GP responsiveness to the changing practice nurse role; and barriers to the expanded role.

\section{Ethics}

The Multi-Region Ethics Committee indicated in writing that formal ethical approval for this project was not required. Assurances were given that interview material would be stored appropriately and that no individual would be identifiable in any of the reports or papers.

\section{Results}

A total of 128 interviews were conducted with an average of eight interviewees in each $\mathrm{PHO}$, including the PHO chair, PHO manager, a Māori, a Pacific, and a community PHO board representative, a PHO board doctor and nurse and a nonboard doctor and nurse. Additional interviews were conducted with the DHB primary care managers, representatives of the Ministry of Health and professional bodies, such as the New Zealand Medical Association, the College of Nurses and the New Zealand Nurses' Organisation, and with 18 nurse leaders. The nurse leaders included representatives from the Nursing Council of New Zealand, the Ministry of Health, the New Zealand Nurses' Organisation and its Primary Health Care Nurses' Council, the College of Nurses and its Primary Health Nursing Network Group, Māori and Pacific provider organisations and academics responsible for PHC nursing programmes.

In addition to the 40 practice nurses, 40 GPs from the practices and the 18 nurse leaders, the majority of the other interviewees were nurses and doctors; three of the nurse leaders were Nurse Practitioners.

Overall, the data from the interviews indicate that there has been substantial growth in a small number of nurses' roles and capability since the introduction of the Strategy. This has mainly occurred with the management of chronic conditions and access to services for underserved and vulnerable groups.

Where nurses' roles have developed, they have been underpinned by two main factors. First, where practices and PHOs have adopted the principles of the Strategy to improve the health of the population, they have expanded the nurses' roles on purpose to increase access to services. Second, where practices and PHOs have taken advantage of additional funding associated with Care Plus, RICF and SIA, nurses have become a resource for increasing practice income. However, although funding has influenced the expansion of some nursing roles, it has acted as a deterrent for others.

\section{Expansion of roles}

Since the introduction of the Strategy where practices have accessed the additional funding and utilised their nurses to provide greater access to services for their patients, some nurses and GPs reported that not only were the nurses providing more services but they were also functioning more autonomously.

We have expanded the nursing staff; we have a Care Plus nurse and an outreach nurse. We have a phone nurse on all the time who triages calls and we have an acute nurse who triages the walk-in acute patients to decide if they need to be seen by the doctor and we have a consulting nurse running a clinic ... we have some specialist clinics ... It works smoothly

$$
(G P)
$$

In larger general practices, where nurses' roles have expanded, they reported that their responsibilities now include: telephone follow-ups; triaging of acute walk-in patients; general consultations; well child consultations; health promotion; and clinics for people with chronic conditions such as diabetes.

Nurses also reported an increase in communitybased services using SIA, RICF and health promotion funding to improve access for patients. These include: one-stop shops for youth; outlying nurse-led clinics; Māori hui; ${ }^{2}$ programmes in schools; community action projects; programmes on

\footnotetext{
${ }^{2} \mathrm{~A}$ hui is a gathering of Māori people.
} 
injury prevention; group safety; marae ${ }^{3}$ safety; water safety; road safety; sexual health; and mobile and evening nurse clinics. In addition, new projects such as 'Get Checked', funded by the Ministry of Health to provide free annual checkups for diabetics (Ministry of Health, 2009c); Care Plus, a PHC initiative for people with two or more chronic conditions; and Youth Health, established in 2007 to increase access for youth to health services (Ministry of Health, 2009f), were all reported to have led to the expansion of nurses' roles.

Where a patient's needs were met by a nurse rather than a GP (eg, diabetes checkups, cervical smears or triage), this was often seen to free up the GP's time, be more cost effective and lead to greater job satisfaction for team members.

One PHO manager, who is also a GP, suggested that nurses are able to provide a large number of PHC services, and that for much of the work there is no need for a doctor.

...the community based medicine that is now being pushed ... is a nurse oriented type of work (PHO manager/GP)

He also reported that his practice had accessed the new funding streams so as to increase the services they offer.

Participants reported that in some areas the expanded role has resulted in increased acceptance of nurses by their communities as their first port of call and people now have more choice about who will provide the services they need. In some practices, the nurses rather than the GPs see patients for follow-up visits. In addition, mainly in the larger practices, nurses are becoming more specialised as, for example, diabetes nurses, asthma nurses and respiratory nurses.

Although the nurse leaders noted some positive developments from the new funding streams, they also questioned the sustainability of some of these initiatives. The funding streams were also thought by some nurse leaders to be fragmenting and divisive of the nursing role, weakening the umbrella identity of the PHC nurse.

\section{Nurse Practitioner role}

Nurse Practitioners were considered to have the potential to significantly improve PHC as they

\footnotetext{
${ }^{3} \mathrm{~A}$ marae is a communal or sacred meeting place for Māori

offer a different level of care and expertise to that of practice nurses. The nurse leaders in particular perceived Nurse Practitioners to be highly effective for providing population-based care such as sexual health and women's health, and for working in remote areas as well as with the aged, with young people in non-traditional settings, in chronic disease case management, with high-risk populations and in primary mental health care. They perceived Nurse Practitioners with their prescribing authority for their speciality areas, and having extensive clinical and contextual knowledge of their speciality areas, to be vital for primary health care. One nurse leader explained:

\section{...the purest manifestation of a Nurse Prac- titioner to me is a primary health care Nurse Practitioner. I don't see them as opposition to GPs or providing a whole alternative service. I see them as individuals who are able to do everything we are advocating for in terms of helping people change their behaviour in the community context \\ (nurse leader)}

Currently, however, the funding arrangements and regulatory restrictions prevent employment opportunities within PHC for Nurse Practitioners and the role reaching its potential. At present, Nurse Practitioners cannot independently enrol patients in a $\mathrm{PHO}$ and therefore need to be employed in general practices. Participants reported that of the few PHC Nurse Practitioners who have registered to date, most have not found appropriate work using their knowledge and skills.

\section{Funding as a barrier to expanding nurses' roles}

Capitation funding to PHOs and practices was intended to shift PHC delivery from an individual patient focus to a population focus. However, there was no requirement for capitation to be used as the mechanism for distributing funding at the practice level, and thus there was not always an incentive at the practice level to adopt a population-based health approach (Croxson et al., 2009).

At the practice level, business incentives are still driving many GPs. GP-owned private businesses can set their own fees for consultations and many 
still focus on patient throughput to maintain their personal income.

We split up the money with a dummy GMS system... we just pretend we are claiming on the old system...

Much of the practice income is still dependent on GPs seeing patients, a major limitation when you are trying to drive lower fees and a multidisciplinary team approach

(PHO manager)

Where fee for service is ongoing there is a powerful incentive not to use nurses

(PHO manager)

The majority of PHOs and general practices where nursing roles had not expanded remained GP-focused and some participants argued that current funding mechanisms are proving to be a disincentive for developing nurses' roles.

... some of our less innovative practices that do not respond to the primary care strategy are also the ones who are charging the high fees and there is no reward for mainstream practices that are using the nurses differently... all the reward is going to the noncompliant practices and all our mechanisms to challenge this are time consuming and ineffective...

\section{(DHB PHC manager)}

There are still practices where the more patients you see the more money you make ... it's a real conflict with you being proactive, doing Care Plus ... Those practices in the old model will not use their nurses ...

(PHO manager)

By not expanding the role of the nurses as intended in the PHCS, the GPs are not only endeavouring to increase their incomes but are denying their patients access to population-based services and choice of provider.

If you can get $\$ 60$ for a smear why have the nurse do it for \$10? They haven't switched

(PHO manager)

A patient comes in to have some stitches outif a nurse saw them it was about $\$ 15$, if a doctor saw them it was about $\$ 32 \ldots$ the doctors are running the business and they do not want to lose out [on] any money

(PHO manager)

Some interviewees considered that greater flexibility with the funding streams would enable practice teams to address and deliver a wider variety of services to their enrolled populations, including domiciliary visits by practice nurses and palliative care nurses:

We could do dressings in the home... we could do that just as well [as district nurses]. We could have our own midwife which would offer a really god service to our patients. It would make us almost completely self-sufficient!

$(P N)$

We need to get back to the community model with midwives, doctors and community nurses working together. We could do a lot more with cardiac and with respiratory...

(PHO Manager)

The nurse leaders had hoped that capitation funding would overturn the existing negative aspects of the employer-employee relationship between GPs and practice nurses and lead to more of a team approach where the most appropriate person would provide the care rather than it being driven by the GPs' need to see as many patients as possible.

Capitation was meant to provide freedom in general practices for practice nurses. It hasn't the GPs employ them and decide what they can do ... Nurses do not decide how nursing services will be provided - GPs do

(Nurse leader)

... as long as the funding is funnelled through the general practitioner and the nurse doesn't have any control over the funding - that's a problem

(Nurse leader)

Some participants suggested that the funding mechanism for PHOs and practices is going to have to change if we are to see the level of teamwork expected within the strategy; it cannot remain funded on a GP register. Nurses as providers within PHOs, they believed, should be able to enrol patients and receive capitation funding for them. 
In pursuit of improved models of working and teamwork, some PHOs have proposed to the DHBs that they should bring practices together into larger centres under a salaried model. This approach was supported by nurse leaders and other participants. They acknowledged that such action would change the working of general practice.

The huge solution out there would be for the PHO to salary doctors and nurses... then there would not be a barrier there...

(Nurse leader)

... unbundle/extricate funding from capitation to enable nurses to be part of a truly autonomous nursing service. To work alongside GPs not be employed by them, and be flexible, accessible and mobile

(Nurse leader)

However, not everyone agreed:

The question of PHOs employing nurses has been raised, but it's not necessarily the only solution. It's one model and may work in some places, but the employer-employee relationship is in my view made too much of when looking at barriers to teamwork

(PN PHO Board member)

There was an acceptance by some PHO members and DHB PHC managers that the potential of the PHCs was not being realised and that considerable changes would have to be made for this to be achieved, but there was not always recognition of how these changes might come about or who would be responsible for them.

There will always be a tension until the GPs see the potential of the PHCS and accept it wholeheartedly, rather than having it forced upon them. There has to be a sea change in both GPs and clients to embrace the wellness model

(Community $\mathrm{PHO}$ representative)

We have not changed as much as we could have ... I do not know if that is us or the patients ... it's still very much doctor orientated ... Patients still come to see us rather than the nurses

\section{(GP, PHO Chair)}

One DHB PHC manager recognised that increasing the role of practice nurses could lead to more efficient ways of working and improve the financial returns for the practices.

So if people have an inefficient model of practice... with a low ratio of nurses to GPs and all those things... if they are inefficient and still charging high fees and want to have a reasonable return in salary, which nobody argues with, ... you cannot say why do you have to charge this when another clinic charges less and still pays the doctors $\$ 120 \mathrm{k}$ a year...

(DHB PHC Manager)

However, not all nurses are comfortable with charging fees for their services. Historically, there have not been charges for nurses' services in general practice, but, within the new environment, there is a growing awareness that this needs to change.

From a nursing perspective, nurses are just beginning to accept that they should be charging for their service. They see this as a way of valuing the nursing service. The future development of nursing needs strong and accountable nurses

(PN PHO member)

\section{Discussion}

Overall, the findings suggest that since the introduction of the Strategy, where practices have either adopted the principles of the Strategy and/ or taken advantage of additional funding associated with managing patients with long-term, chronic conditions and improving access, there has been substantial growth in the development of some nurses' roles and these nurses, mainly employed in larger practices, are working more autonomously. Hefford et al. (2010) found that general practices could be more efficient if they used their nurses more and charged at least 50\% of the GPs' consultation fee for their services.

As Smith (2009) found, the additional funding sources have not proved to be a sufficient incentive for the majority of practices to significantly change the ways they work or increase the services they offer by expanding the role of their nurses.

Similar to the positive changes that have taken place for the minority of practice nurses in NZ, international literature reports expansion of PHC nurses' roles in countries such as Australia, 
Canada, England and the United States (Buchan and Calman, 2005). Development has occurred in the role and scope of Australian practice nursing in the last decade due to the growing need for chronic and complex disease management and provision of preventive health care in the community (Halcomb et al., 2006).

In England, additional funding provided to GPs to improve the quality of PHC through the Quality Outcomes Framework (QOF), a complex set of quality indicators, has seen practice organisation and nurses' responsibilities change substantially. Patients are now being seen more frequently in disease-oriented clinics run by nurses and nearly all routine care for chronic diseases specified in the QOF is being delivered by nurses (Roland et al., 2006; Maisey et al., 2008).

International literature indicates that barriers to the expansion of nursing roles in NZ are similar to those in England, Canada, the United States and Australia. These include regulatory and funding issues related to the employment of Nurse Practitioners and the continued employment of practice nurses by GPs (Perry et al., 2005; Gardner et al., 2007).

Although specific funding schemes have resulted in some NZ nurses expanding their activities and offering services to under-served populations and people with high needs, the current funding model, inconsistent uptake of specific funding initiatives by some practices, related high transaction costs and the short-term nature of some programme contracts (eg, RICF, SIA and Care Plus) have limited their overall impact, such that change is not occurring at the same level nationwide. The inconsistent, piecemeal uptake of this element of the Strategy has resulted in patients having less choice of provider and fewer services available to them, especially services focussed on managing long-term conditions and those focussed on improving access for under-served groups.

The Strategy was an open-ended document that allowed for evolutionary change. This has resulted in a large variation in the size and structure of PHOs and their underpinning philosophies, which have inevitably impacted on health-care professionals and practices and the way services are provided. Although the PHOs are funded on a capitation basis, funding at the practice level, where services are provided, is in many cases still dependent on the number of patients a GP sees, including patient co-payments, which are set by individual practitioners and practices. This is neither conducive to innovative approaches and collaborative working arrangements nor does it provide an incentive for practices to have their nurses working more autonomously.

Raymont and Cumming (2009) found that $90.5 \%$ of the 277 GPs in their study reported that the two factors influencing the distribution of income to GPs who were either owners or partners in a practice were the number of consultations and the volume of co-payments. This is a strong disincentive for expanding nurses' roles and thus expanding services and choice of provider.

Fundamentally, the new arrangements have continued the juxtaposition of public funded PHOs and private enterprise general practice. For many practices, GPs' incomes are still dependent on their number of patient visits. The changes to the funding of PHC services, therefore, have not gone far enough.

The policy underpinning the Strategy did not require changes at the practice level but assumed that the capitation funding and additional funding streams would provide sufficient incentives for practices to develop a stronger team approach and, consequently, changes in the way PHC services are delivered. What the policy did not take into account was the private enterprise business model of the majority of practices. On the other hand, it could be argued that the then government devolved responsibility for bringing GPs on board to the PHOs as the national-led government in the early 1990s had done with the Regional Health Authorities (RHAs). As with the PHOs, the RHAs were made responsible for contracting with the GPs to ensure that their populations received appropriate PHC services. At that time, the GPs banded together to form Independent Practitioner Associations (IPAs) to contract on their behalf and they were able to maintain their existing ways of providing services. Some of those IPAs have since become PHOs with very little change to their structures and goals.

However, by not ensuring sufficient support for the goals of the policy by those responsible for implementing it at the practice level, successful implementation at a national level could not be guaranteed. Few practices have adopted the principles of population-based health care and reducing inequalities by offering more teambased and appropriate services. The majority 
have continued with GP-driven, fee-for-service, individually focussed care.

\section{Conclusion}

To ensure that the goals of a policy are realised, in this instance the Strategy, it is important that those responsible for implementing it at the coalface are sympathetic to the policy intentions. In the case of the Strategy, the previous government assumed that capitation funding and additional funding streams would provide sufficient incentives for GPs to change the ways they practise, from treating individuals experiencing illness and accidents to taking a population-based approach to providing care, the latter requiring them to re-focus their way of working to include a more systematic approach to disease management that also includes disease prevention and health promotion. This requires a team approach and nurses were to be a crucial part of the team expanding their current roles to provide increased access to appropriate services. By not taking into account the business model of the majority of general practices that are based on income generation, the previous government was not able to achieve all the intentions of the strategy.

The current government has the opportunity to better incentivise GPs to take this broader approach, and this will become critical with the increasing burden of long-term conditions that need to be managed in PHC.

\section{Acknowledgements}

The authors received financial support for the research from the New Zealand Health Research Council, Ministry of Health and Accident Compensation Corporation. The authors have no conflict of interest with respect to the authorship and/or publication of this article.

\section{References}

Armstrong, F. 2005: What lies ahead for primary care? Australian Nursing Journal 13, 18-21.

Bodenheimer, T. 2003: Primary care in the United States: Innovations in primary care in the United States. British Medical Journal 326, 796-98.

Primary Health Care Research \& Development 2012; 13: 120-129
Buchan, J. and Calman, L. 2005: Skill-mix and policy change in the health workforce: nurses in advanced roles OECD Health Working Paper no. 17. Paris: OECD.

Carnwell, R. and Daly, W.M. 2003: Advanced nursing practitioners in primary care settings: an exploration of the developing roles. Journal of Clinical Nursing 12, 630-42.

Croxon, B., Smith, J. and Cumming, J. 2009: Patient fees as a metaphor for so much more in New Zealand's Primary Health Care System. Wellington: Health Services Research Centre.

Department of Health. 2006: Our health our care our say: a new direction for community services. London: The Stationery Office.

Dodoo, M., Roland, M. and Green, L. 2005: UK lessons for US primary care. Annals of Family Medicine 3, 561-62.

Finlayson, M., Sheridan, N. and Cumming, J. 2009: Nursing developments in Primary Health Care 2001-2007. Wellington: Health Services Research Centre.

Gardner, G., Chang, A. and Duffield, C. 2007: Making nursing work: breaking through the role confusion of advanced practice nursing. Journal of Advanced Nursing 57, 382-91.

Halcomb, E.J., Patterson, E. and Davidson, P.M. 2006: Evolution of practice nursing in Australia. Journal of Advanced Nursing 55, 376-88.

Hefford, M., Cumming, J., Finlayson, M., Raymont, A., Love, T. and Van Essen, E. 2010: Practice nurse cost benefit analysis: report to the Ministry of Health. Wellington: LECG.

Kent, B., Horsburgh, M., Lay-Yee, R., Davis, P. and Pearson, J. 2005: The National Primary Medical Care Survey: report 9, nurses and their work in primary health care. Auckland: University of Auckland.

King, A. 2001: The primary health care strategy. Wellington: Ministry of Health.

Maisey, S., Steel, N., Marsh, R., Gillam, S., Fleetcroft, R. and Howe, A. 2008: Effects of payment for performance in primary care: qualitative interview study. Journal of Health Services Research \& Policy 13, 133-39.

Ministry of Health. 2009a: Care Plus. Retrieved April 2009 from http://www.moh.govt.nz/moh.nsf/indexmh/phcs-funding-careplus.

Ministry of Health. 2009b: First Contact. Retrieved April 2009 from http://www.moh.govt.nz/moh.nsf/indexmh/phcs-fundingfirstcontact.

Ministry of Health. 2009c: Getting Checked. Retrieved April 2009 from http://www.moh.govt.nz/moh.nsf/indexmh/phcsfunding-getchecked.

Ministry of Health. 2009d: Primary Health Care Funding. Retrieved April 2009 from http:/www.moh.govt.nz/ moh.nsf/indexmh/phcs-funding.

Ministry of Health. 2009e: Primary Health Organisations. Retrieved April 2009 from http://www.moh.govt.nz/ moh.nsf/indexmh/phcs-pho.

Ministry of Health. 2009f: Youth Health. Retrieved April 2009 from http://www.moh.govt.nz/moh.nsf/indexmh/youthhealthcurrentwork. 
Nursing Council of New Zealand. 2011: Nurse Practitioner scope of practice. Retrieved June 2011 from http://www. nursingcouncil.org.nz/download/74/np_stats_24june11.pdf

Perry, C., Thurston, M., Killey, M. and Miller, J. 2005: The nurse practitioner in primary care: alleviating problems of access? British Journal of Nursing 14, $255-259$.

Primary Health Care Nurse Innovation Evaluation Team. 2007: The evaluation of the eleven primary health care nursing innovation projects: a report to the Ministry of Health by the Primary Health Care Nurse Innovation Evaluation Team. Wellington: Ministry of Health.
Raymont, A. and Cumming, J.M. 2009: Status and activities of general medical practices. Wellington: Health Services Research Centre.

Roland, M., Campbell, S., Bailey, N., Whalley, D. and Sibbald, B. 2006: Financial incentives to improve the quality of primary care in the UK: predicting the consequences of change. Primary Health Care Research \& Development 7, 18-26.

Smith, J. 2009: Critical analysis of the implementation of the Primary Health Care Strategy implementation and framing of issues for the next phase. Wellington: Ministry of Health.

Thomas, D.R. 2006: A general inductive approach for analyzing qualitative evaluation data. American Journal of Evaluation 27, 237-46. 